\title{
Justice evaluations and just earnings: Analysis of a pilot study
}

\author{
Kinga Wysieńska-Di Carlo Z Zbigniew Karpiński, $\mathrm{PhD}$
}

April 24, 2019

\section{Setting the stage: one rater and one rewardee}

Say somebody - let's call them "Person" - comes to you and asks how much you think they should be paid for their job, but instead of asking this question directly they show you ( $a$ ) their resume with a summary of their education, qualifications, and job experience; $(b)$ a short job description with a summary of tasks typically performed at the job; and (c) some additional information concerning their occupational and family circumstances. Now, after you've taken some time to carefully review the material, Person asks you: "Say my net monthly earnings in this job are 1,000 monetary units (MUs). Would you say this amount would be appropriate, given the information you have about me and the job?" You're also told to answer this question using numbers ranging from, let's say, -5 to +5 , with negative numbers corresponding to the idea that the earnings are too low (relative to some reference point) and positive - to the idea that they're too high. Also, the more the number you give as the answer departs from zero (in either direction), the more you find the particular amount to be inappropriate. So, you think for a while and produce a number that we denote by $j_{1}$. Thus, to the first (hypothetical) amount of earnings there corresponds your evaluation of how appropriate this amount is, so we can record our first measurement outcome as a pair $\left(a_{1}, j_{1}\right), a_{1}$ being the first amount. Then, Person asks: "And if I earned 12,450 MUs? How appropriate would that be, according to you?" Again, you give a number in response and we have another pair of values as our second measurement outcome, $\left(a_{2}, j_{2}\right)$. Then Person comes up with another hypothetical amount of earnings and you provide yet another answer, giving us a third measurement, $\left(a_{3}, j_{3}\right)$. This procedure continues for a while, resulting in a series of pairs of values. In each pair, one element represents the hypothetical amount of earnings and the other - your assessment of how appropriately the amount would reward the person's work.

An implicit assumption underlying this little exercise is that, once you have received sufficient information about Person, you will be able to come up with an idea as to how much Person should earn in order for the earnings to be a fair pay for Person's job. Furthermore, it is precisely this idea that "drives" your assessments of the hypothetical amounts suggested by Person. You may not be able to say what the fair pay is exactly, but you have a sense that a particular amount is close to or distant from it. In other words, you come to view a particular amount as more or less appropriate, because it comes more

Institute of Philosophy and Sociology of the Polish Academy of Sciences, Warsaw, Poland. Direct correspondence to zbigniew.karpinski@startmail.com. Research reported in this note is supported by a grant from the National Science Centre awarded to Dr. Kinga Wysieńska-Di Carlo; the title of the grant is Status, fairness, and legitimacy of inequality. Gender and parenthood effects on perceptions of just incomes. 
or less close to what you believe would be fair. Similarly, you come to view an amount as inappropriate, because it is far removed from what you think would be fair. Since the reference point for your assessments is your idea of Person's just earnings, the assessment are really justice evaluations - and we shall refer to them as such from now on.

Given the hypothetical amounts of actual earnings, and your evaluations of these amounts, we can "retrieve" your idea of the fair pay for Person, using the following justice evaluation function proposed by Guillermina Jasso (2006):

$$
J=\theta \ln \frac{A}{C}
$$

In that formula, $J$ is a variable representing justice evaluations you make, $A$ is a variable representing the hypothetical amounts of just earnings read out by the person, and $C$ is the idea of fair pay; unlike $J$ and $A, C$ is a constant - because you can only have one idea of a fair pay for a given individual. The parameter $\theta$ is also a constant which has a special meaning in comparison theory. Jasso (2006) labelled the parameter "signature constant" and defined it as a measure of individual sensitivity to injustice. More specifically, the same departure of actual earnings from the fair amount can be evaluated differently by different people, with some actors voicing their concerns privately and others joining organised collective action to challenge the injustice. Greater absolute values of the signature constant indicate greater sensitivity to injustice, whereas its sign indicates whether the good is perceived by the subject as a reward or burden, with $\theta>0$ signifying the former. Given that our example is about you making evaluations of Person's earnings and that on-the-job earnings are most certainly a reward, we can safely assume $\theta$ to be positive.

The signature constant and the logarithmic transformation both "translate" the departure of $A$ from $C$ into a justice evaluation. The evaluation $J$ assumes negative values if you feel the person is underrewarded, i.e., if their actual payoff, $A$, is below the fair pay, $C$, and it assumes positive values you feel the person is overrewarded. Also, $J=0$ is the point of perfect justice where actual payoff matches the comparison standard exactly. The justice evaluation function has a number of appealing properties, both formal and substantive, than have been discussed extensively in the literature; we encourage readers to consult that literature for more details concerning these properties.

Let us now rewrite Eq. 1 a little to highlight the fact that the amounts of earnings you evaluate and the justice evaluations you make about them are variable, while all other quantities in that formula are constants:

$$
j_{i}=\theta \ln \frac{a_{i}}{C},
$$

where the subscript ranges from 1 to $m, m$ being the number of amounts you assess. Rearranging terms yields:

$$
j_{i}=-\theta \ln C+\theta \ln a_{i}
$$

Notice that $\left\{a_{i}\right\}$ and $\left\{j_{i}\right\}$ are known to us as outcomes of the measurement procedure described above. Other quantities are unknown, however, and have to be estimated from the data. In order to obtain these estimates, we need to find an appropriate statistical model for the data; by contrast, the formula in (2) provides a theoretical model. In our case, given the functional form of (2), the statistical model in question is simple linear regression:

$$
y_{i}=\beta_{0}+\beta_{1} x_{i}+\epsilon_{i}
$$

In (3), $\beta_{0}$ is a constant corresponding to $-\theta \ln C$ in (2). Similarly, $\beta_{1}$ is a constant corresponding to $\theta$, whereas $\left\{y_{i}\right\}$ and $\left\{x_{i}\right\}$ are variables corresponding to $\left\{j_{i}\right\}$ and $\left\{\ln a_{i}\right\}$, respectively. Fitting the regression model to our data, we can obtain estimates of $\theta$ and $C$, because

$$
\hat{\theta}=\hat{\beta}_{1} \text { and } \ln \hat{C}=-\hat{\beta}_{0} / \hat{\beta}_{1}
$$


Exponentation of the latter quantity will produce an estimate of $C$, your idea of just earnings for Person.

\section{Multiple raters, multiple rewardees}

So far in our story, two elements have been kept constant - and we do not mean the constants in the formulas above - namely, (a) you are the only rater making justice evaluations; ( $b$ ) Person is the only rewardee whose earnings are being assessed. But the whole setting can be extended to include (a) multiple raters, or subjects making evaluations of other peoples' earnings; (b) multiple rewardees whose earnings are being assessed. ${ }^{1}$ We used this design in a recently conducted study. In that study, we sampled a group of $n=200$ respondents from a larger pool and gave each respondent a set of vignettes, or standardised descriptions of fictitious individuals in terms of

- gender,

- age - with 8 levels, ranging from 30 to 65 in five year increments,

- occupation - with 21 occupational titles selected from the Polish Social Classification of Occupations (Domański, Słomczyński, and Sawiński 2009) and varying in terms of prestige, ${ }^{2}$

- job experience (in years), and

- the number of children they have, ranging from 0 to 4.

Thus, each respondent in our sample was a rater and each vignette described one rewardee. The vignettes were sampled randomly from a population of vignettes which had been prepared earlier by crossing all levels of a given characteristic with all levels of all the other characteristics. More specifically, the vignettes were sampled from the vignette population using a stratified design. The basis of the stratification was occupation and only one element (i.e., employee) was sampled from each stratum. Thus, because we used 21 occupational titles in designing the vignettes, each respondent received 21 vignettes to evaluate. Also, because a vignette sample was selected for each respondent separately, the same vignette could be assigned to more than one respondent.

Further, we also randomly assigned six hypothetical amounts of earnings to each vignette and asked respondents how appropriate they thought each such amount would be for a person described in the particular vignette. Thus, because each respondent received 21 vignettes to evaluate and because there were six hypothetical amounts per vignette, each subject made a total of 126 justice evaluations. Hence, in order to appropriately represent the structure of our data, we need to rewrite earlier formulas a little:

$$
j_{g h i}=\theta_{i} \ln \frac{a_{g h i}}{C_{h i}}
$$

In this equation, $a_{g h i}$ stands for the $g$-th amount assigned to the $h$-th vignette given to the $i$-th respondent; $j_{g h i}$ denotes justice evaluation made by respondent $i$ about amount $g$ assigned to vignette $h$. The

1. This is an instance of what Jasso (2007) labels "multiple-reward-per-rewardee design".

2. We used the following occupational titles (occupational prestige scores in parentheses): professor of early education (97.3), anesthesiologist (87.8), actor (86.9), radio news presenter (81.2), plane pilot (80.6), cloakroom attendant (8.6), it security specialist (77.5), artistic director in an advertisement agency (72.7), animal technician (66.1), librarian (61.3), train dispatcher (60.9), nurse (55.9), customs officer (54.9), real estate agent (51.4), accountant (50.9), cashier in a supermarket (27.6), mailperson (27.5), waitperson (25.7), hairdresser (21.2), telemarketer (18.0), janitor (10.4). 
term $\theta_{i}$ represents respondent-specific signature constant and $C_{h i}$ refers to respondent $i$ 's idea of a fair pay for employee $h$. Note that, unlike in the previous example, the signature constant is allowed to vary from respondent to respondent. The same applies to $C$ : instead of being constant, it is allowed to very between respondent-vignette (or rater-rewardee) combinations.

After appropriate algebraic manipulations, the last formula expands to:

$$
j_{g h i}=-\theta_{i} \ln C_{h i}+\theta_{i} \ln a_{g h i}
$$

Again, $\left\{a_{g h i}\right\}$ and $\left\{j_{g h i}\right\}$ are known to us, because we either explicitly varied or measured them in our study, but all other elements of Eq. (4) have to be estimated from our data. As previously, we need an appropriate statistical model to obtain the estimates. Note that simple linear regression is no longer appropriate for the purpose of estimating (4), because in a simple regression model parameters $\beta_{0}$ and $\beta_{1}$ are fixed, whereas we are now searching for a model that would allow these parameters to vary. That is, while the relationship between $\left\{j_{g h i}\right\}$ and $\left\{a_{g h i}\right\}$ is still assumed to be linear, simple linear regression would not be sufficient to model the data, as it doesn't allow the intercept to vary over rater-rewardee combinations and the slope - across raters. This is why we resort to linear mixed-effects models, as they permit for the variability in the parameters to be explicitly introduced into the model.

Linear mixed-effects models are suitable whenever obervations are "grouped", as they often are in cross-national, clinical, or educational research. In cross-national studies, respondents are grouped by country; in clinical studies, patients are grouped by hospitals, which in turn are grouped by district; in educational research, students are grouped by schools, schools — by jurisdictions and jurisdictions by municipalities. And so on, and so forth. In our study, observations (i.e., justice evaluations) are also grouped: by respondents and by vignettes. Unlike in the clinical or educational research, groups based on one factor are not "nested" in groups based on the other. That is, in the case of an educational survey of a representative sample of students it makes sense to say that students are nested within schools and schools are nested jurisdictions, but it would not make sense to say that in our study vignettes were nested within respondents, because, as mentioned above, the same vignette could be assigned to different respondents. For this reason, we say that the random effects of respondents and vignettes are crossed rather than nested.

A linear mixed-effects model for Eq. (4) has the following form:

$$
\begin{aligned}
y_{g h i} & =\pi_{0 h i}+\pi_{1 i} x_{g h i}+\epsilon_{g h i} \\
\pi_{0 h i} & =\beta_{0}+u_{0 h}+u_{0 i} \\
\pi_{1 i} & =\beta_{1}+u_{1 i}
\end{aligned}
$$

In this model, $\beta_{0}$ and $\beta_{1}$ are fixed parameters and are interpreted as the intercept and slope, respectively, as in in "ordinary" linear regression. Further, $\left\{u_{0 h}\right\}$ are deviations from the intercept which represent random effects of vignettes. Similarly, $\left\{u_{0 i}\right\}$ are departures from the intercept that represent random effects of respondents. Finally, $\left\{u_{1 i}\right\}$ represent variability of respondent-specific slopes around $\beta_{1}$. Making substitutions and rearranging terms gives:

$$
y_{g h i}=\underbrace{\beta_{0}+\beta_{1} x_{g h i}}_{\text {fixed part }}+\underbrace{u_{0 h}+u_{0 i}+u_{1 i} x_{g h i}+\epsilon_{g h i}}_{\text {random part }}
$$

In the next section, we fit this model to the data from our study using function textttlmer from package lme4 (Bates et al. 2015) in the R Environment for Statistical Computing (R Core Team 2017). 


\section{Estimating the model parameters}

As a first step, let us have a look at the small subset of the data that we are using in this analysis. The output below tell us that there are 25,200 observations in the data set, which corresponds to justice evaluations made about each of the six earnings amounts assigned to each of the twenty-one vignettes assigned to each of the two hundred participants in the study. We also learn from the output that the first vignette assigned to the participant with ID number 10 has ID 84. The first amount assigned to that vignette is equal to 1,900 PLN and it received the evaluation of -5 , the lowest possible on a scale ranging from -5 to 5 .

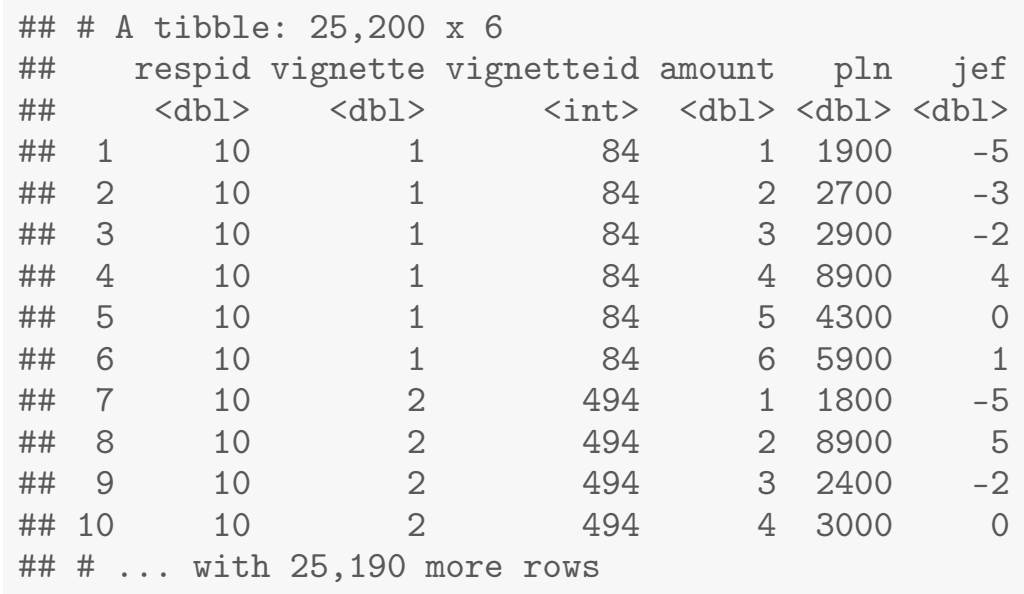

\subsection{Random intercept models}

The first model that we fit to the data is a random-intercept model with random effects of both respondents and vignettes:

fit_0<- $\operatorname{lmer}($ jef $\sim 1+(1$ | respid $)+(1$ | vignetteid), data = p)

This is an "empty" model, i.e., a model with an intercept only and no predictors, as signified by the " 1 " following the tilde in the model formula above. The expressions ( 1 |respid) and ( 1 |vignetteid) indicate that the intercept is allowed to vary across respondents and vignettes, respectively. Also, there is a separate term for each grouping factor, because of the "crossed" character of the random effects, as explained earlier. We are interested in the variance of the random effects and specifically - in the proportion of the total variance of the dependent variable that can be attributed to each grouping factor. The results are shown in Table 1.

As we can see, each of the grouping factors contributes more or less equally to the total variance of the dependent variable (i.e., justice evaluations; see Model 1 in Table 1). Specifically, the estimated variance of the random effects associated with respondents is 0.713 , which accounts for $/(0.713+0.707+7.92)=$ 0.0763 of the total variance. In other words, as much as $7.63 \%$ of the total variance in justice evaluations can be attributed to differences among respondents. 
Table 1: Parameter estimates for the baseline random-intercept models

\begin{tabular}{lccc}
\hline & Model 1 & Model 2 & \multicolumn{1}{c}{ Model 3 } \\
\hline Constant & 0.034 & 0.034 & 0.021 \\
& $(0.068)$ & $(0.036)$ & $(0.063)$ \\
$\operatorname{Var}\left(u_{0 h}\right)$ & 0.713 & 0.886 & \\
$\operatorname{Var}\left(u_{0 i}\right)$ & 0.707 & & 0.725 \\
$\operatorname{Var}\left(\epsilon_{g h i}\right)$ & 7.924 & 8.475 & 8.624 \\
& & & \\
\hline Groups - vignetteid & 981.000 & 981.000 & \\
Groups - respid & 200.000 & & 200.000 \\
\hline Log-likelihood & -62634.678 & -63296.923 & -63150.224 \\
\hline \multicolumn{1}{c}{ Significance: $* * * \equiv p<0.001 ; * * \equiv p<0.01 ; * \equiv p<0.05$}
\end{tabular}

Significance: $* * * \equiv p<0.001 ; * * \equiv p<0.01 ; * \equiv p<0.05$

In order to see if the variance due to respondents is statistically significantly different from 0 , we compared our baseline model with a simpler variant that contains only the random effect of vignettes (see Model 2 in Table 1). The test statistic is 1,323; it follows chi-square distribution based on $d f=1$. The result is highly significant, well beyond the standard significance level of 0.05 . We can therefore conclude that the variance component due to differences between respondents is statistically significant. Estimate of the variance of the random effects associated with vignettes is interpreted in a similar manner.

\subsection{Fixed and random slope models}

As a next step, let us extend the model by adding to it a fixed-effect of the log of the amounts evaluated by the respondents, i.e., $\ln a_{g h i}$, as specified by Eq. (4). The model is fit to our data using the following command:

fit_1 <- $\operatorname{lmer}($ jef $\sim \log (\mathrm{pln})+(1 \mid$ respid $)+(1 \mid$ vignetteid $)$, data $=$ p)

Parameter estimates for this model are shown in Table 2 (see Model 4). As we can see, the estimate of residual variance for that model is 2.19 , or $2.19 / 7.92=27.6 \%$ of the residual variance in the baseline model. This is to say that adding the log of actual earnings to the model reduces the residual variance by $72.4 \%$. In still other words, $72.4 \%$ of the variance that is not attributable to differences among respondents or differences between vignettes is accounted for by the differences in the amounts of earnings assigned to each vignette. To illustrate, according to the estimates, doubling the amount that is being evaluated is predicted to result in moving the justice evaluation for that amount up by $\hat{\beta}_{1} \cdot \ln 2=$ $4.09 \cdot 0.693=2.83$ points on the justice scale. Similarly, halving the amount results in shifting the evaluation down by the same magnitude. In general, multipling an amount by $k$ changes the evaluation by $4.09 \mathrm{k}$ points.

While $72.4 \%$ reduction in the residual variance may seem like lot, it could be argued that, given the design of our study, it is actually surprisingly low. That is, because the only element affecting jus- 
Table 2: Parameter estimates for the models with the log of actual earnings as a predictor

\begin{tabular}{lcc}
\hline & Model 4 & Model 5 \\
\hline Constant & $-33.850^{* * *}$ & $-33.919^{* * *}$ \\
Log of actual earnings & $\begin{array}{c}(0.152) \\
\left(0.089^{* * *}\right.\end{array}$ & $\begin{array}{c}4.098^{* * *} \\
(0.016)\end{array}$ \\
$\operatorname{Var}\left(u_{0 h}\right)$ & 0.838 & 0.846 \\
$\operatorname{Var}\left(u_{0 i}\right)$ & 0.761 & 57.833 \\
$\operatorname{Var}\left(\epsilon_{g h i}\right)$ & 2.189 & 1.915 \\
$\operatorname{Var}\left(u_{1 i}\right)$ & & 0.804 \\
$\operatorname{Cov}\left(u_{0 i}, u_{1 j}\right)$ & & -6.777 \\
& & \\
\hline Groups - vignetteid & 981.000 & 981.000 \\
Groups - respid & 200.000 & 200.000 \\
\hline Log-likelihood & -47110.534 & -45784.900 \\
\hline Significance: $* * * \equiv p<0.001 ; * * \equiv p<0.01 ; * \equiv p<0.05$
\end{tabular}


tice evaluations about a particular vignette - once the variance associated with differences between respondencts and differences between vignettes has been accounted for - are the amounts of earnings that were randomly assigned to it, one may expect the percentage of variance reduction to be much closer to 1 . The relatively low proportion of reduction in variance could be a sign of inaccuracy of the justice-evaluation function proposed by Jasso (2006) or some feature of our study that interacts with respondents' behaviour (see Eriksson 2012, for a related point).

In Model 4, the effect of the (hypothetical) amounts of actual earnings is fixed. But, as explained above, estimating the amounts of just earnings for each respondent-vignette combination, requires the effect of actual earnings to vary across respondents, as specified in Eq. (4). This calls for a model with random slopes. In order to fit that model to our data, we have to rewrite the previous command as follows:

fit_2 <- $\operatorname{lmer}(j e f \sim \log (\mathrm{pln})+(1+\log (\mathrm{pln}) \operatorname{lrespid})+(1 \mid$ vignetteid $)$, $\operatorname{data}=\mathrm{p})$

Parameter estimates for the random-intercept and random-slope model are also presented in Table 2 (under the header "Model 5"). Let us first check if the variability in slopes is sufficiently large to justify the use of the random-slope effects. We do so by comparing log-likelihoods of the models with and without random slopes. The test statistic equals $-2(-47,111+45,785)=2,651$ based on $d f=$ 2 , giving $p \ll 0.05$. Note that the test is based on two degrees of freedom, because in fitting the random-intercept and random-slope model two additional parameters are estimated, relative to the model without random slopes, namely, $(a)$ the variance in respondent-specific slopes, $\operatorname{Var}\left(u_{1 j}\right)$ and (b) the covariance between respondent-specific intercepts and respondent-specific slopes, $\operatorname{Cov}\left(u_{0 i}, u_{1 i}\right)$. As we can see, the covariance is negative, implying that respondents with higher estimated slopes have lower estimated intercepts. Substantively, this result is quite appealing, given the interpretation of slope for $\ln a_{g h i}$ in terms of sensitivity to injustice. That is, the negative estimate of $\operatorname{Cov}\left(u_{0 i}, u_{1 i}\right)$ means that respondents who are more sensitive to injustice have lower estimated intercepts. Further, because the intercept indicates "baseline" justice evaluation when $\ln a_{g h i}=0$ or $a_{g h i}=1$, the lower the intercept, the more "outrageous" the proposition that actual earnings be equal to $1 \mathrm{MU}$ is perceived to be. In other words, the negative covariance of random effects associated with respondents implies that respondents who are more sensitive to injustice find the proposition that actual earnings be equal to $1 \mathrm{MU}$ more outrageous.

This is illustrated in Figure 1 which graphs estimated justice-evaluation functions for two "extreme" individuals: the one with the highest and the one with lowest estimated sensitivity to injustice. That is, the lines shown in the graph represent changes in the justice evaluations made by these respondents as a function of the logged amounts of actual earnings. As we can see, the line for the more sensitive respondent is much steeper than for the less sensitive one. The lowest amount assigned to vignettes in our study was equal to $1,800 \mathrm{PLN}$; the $\log$ of that amount is equal to $\ln 1,800=7.5$. Even though both subjects view this amount as unfairly low (both lines are below zero at $x=7.5$ ), the one who is more sensitive finds it much more unfair than the other respondent. Similarly, the highest amount of earnings that we asked our subjects to evaluate was equal to 13,300 PLN, corresponding to $\ln 13,300=9.5$ on the log scale. Again, we see that both lines are above zero at this point, meaning that both respondents view this amount as unfairly high, but while the less sensitive one views it as a very mild over-reward, the other person perceives the unfairness as being a few orders of magnitude higher. 
Figure 1: Estimated random slopes for two "extreme" cases

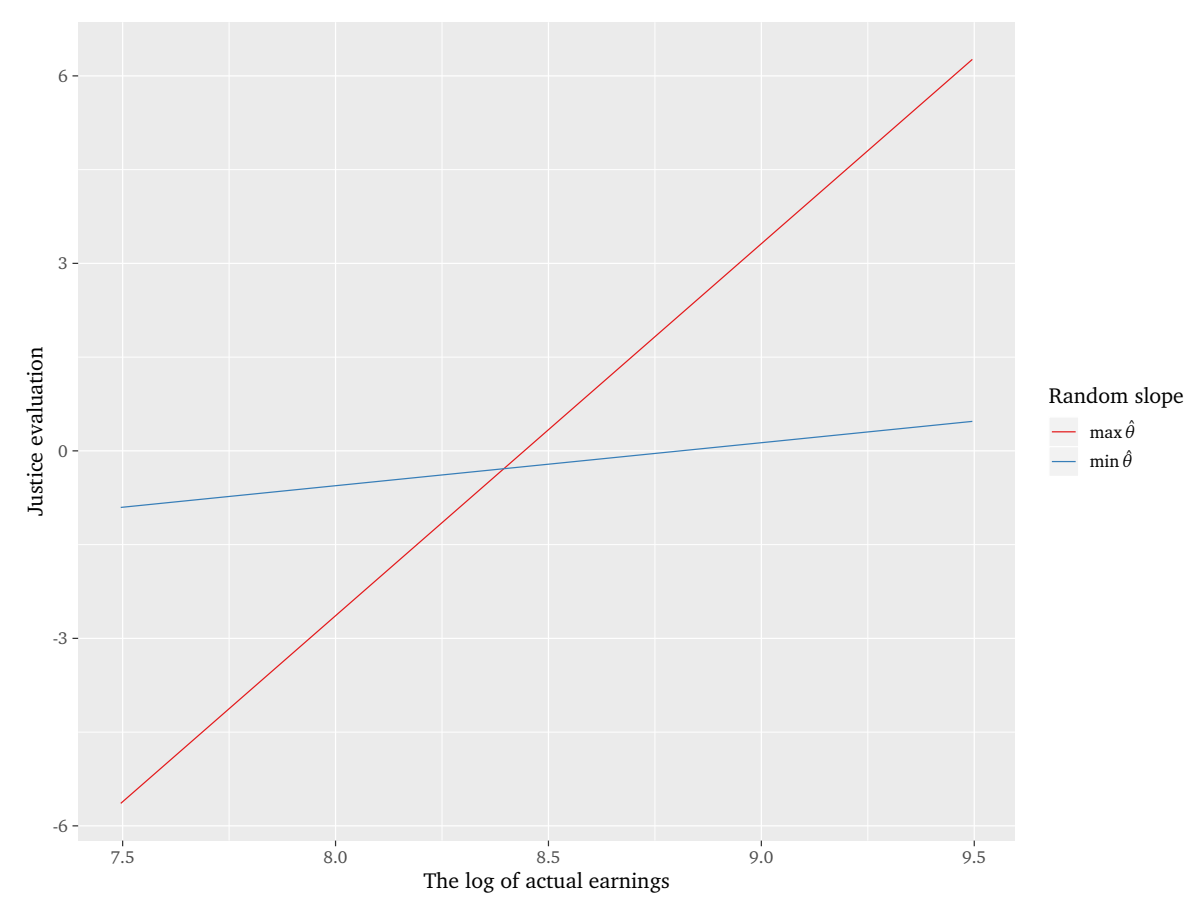




\subsubsection{Variance decomoposition in the random slope model}

Returning to the estimates of the random effects in Model 5 in Table 2, because there are two types of random effects associated with respondents - random intercepts and random slopes - the total amount of variation that is attributable to differences between respondents is given by: ${ }^{3}$

$$
\begin{aligned}
\operatorname{Var}\left(u_{0 i}+u_{1 i} x_{g h i}\right)=\operatorname{Var}\left(u_{0 i}\right)+2 \operatorname{Cov}\left(u_{0 i}, u_{1 i} x_{g h i}\right) & +\operatorname{Var}\left(u_{1 i} x_{g h i}\right)= \\
= & \operatorname{Var}\left(u_{0 i}\right)+2 \operatorname{Cov}\left(u_{0 i}, u_{1 i}\right) x_{g h i}+\operatorname{Var}\left(u_{1 i}\right) x_{g h i}^{2}
\end{aligned}
$$

So, the variance of the random effects associated with respondents turns out to be a quadratic function of $x_{\text {ghi }}$, i.e., the log of the actual earnings amounts evaluated by the study participants. This function is minimised when $x_{g h i}=-\operatorname{Cov}\left(u_{0 i}, u_{1 i}\right) / \operatorname{Var}\left(u_{1 i}\right)=6.78 / 0.804=8.42$. That is, the random effects associated with respondents show the least variability when the amount of actual earnings is $\exp 8.42=4,555$ PLN. Further, notice that substituting $x_{g h i}$ into Eq. (7) reduces the formula to just $\operatorname{Var}\left(u_{0 i}\right)$, implying that the estimate of $\operatorname{Var}\left(u_{0 i}\right)$ for the model is the estimated variance of the random terms associated with respondents when $x_{g h i}=0$, i.e., when $a_{g h i}=1$. But otherwise the estimate is not meaningful in itself and has to be interpreted together with other random effects for that model. Specifically, recall that the lowest amount of earnings that our subjects were given to evaluate was equal to 1,800 PLN. Thus, the proportion of the variability the random effects that is attributable to respondents when $x_{g h i}=\ln 1,800$ is, by (7)

$$
\frac{\operatorname{Var}\left(u_{0 i}\right)+2 \operatorname{Cov}\left(u_{0 i}, u_{1 i}\right) \ln 1,800+\operatorname{Var}\left(u_{1 i}\right)(\ln 1,800)^{2}}{\operatorname{Var}\left(u_{0 h}\right)+\operatorname{Var}\left(u_{0 i}\right)+2 \operatorname{Cov}\left(u_{0 i}, u_{1 i}\right) \ln 1,800+\operatorname{Var}\left(u_{1 i}\right)(\ln 1,800)^{2}+\operatorname{Var}\left(\epsilon_{g h i}\right)},
$$

which after appropriate substitutions gives 0.343 . That is, as much as $34.3 \%$ of the variance when $a_{g h i}=1,800$ PLN can be attributed to differences between respondents with respect to how they evaluate this amount. Similarly, the highest amount of earnings that we asked our study participants to evaluate was equal to 13,300 PLN. The proportion of the variance that is attributable to differences between respondents when $a_{g h i}=13,300$ PLN is, by the same token, 0.377 . So, we can see that the proportion of variance is somewhat higher at the higher amount. This is also illustrated by Figure 2 which graphs the variance function in Eq. (7) for the actual earnings ranging from 1,800 PLN to 13,300 PLN, as in our study.

\section{Retrieving the just earnings amounts}

In this section, we use the parameter estimates from the random-intercept and random-slope model to obtain the estimates of just earnings for each respondent-vignette combination. To do so, we first need to "retrieve" all the random effects from the model and merge them with the original data. This way, we'll be able to calculate the respondent-specific slopes and repondent-vignette-specific intercepts. Then, dividing the negative of the latter by the former, we'll get our estimates of the log of just earnings, as explained earlier.

The following commands "extract" the random effects of vignettes from the model:

3. This formula is taken from the materials published at University of Bristol's Centre for Multilevel Modelling website, especially the presentations on random slope models. 
Figure 2: Plot of the variance function in Eq. (7)
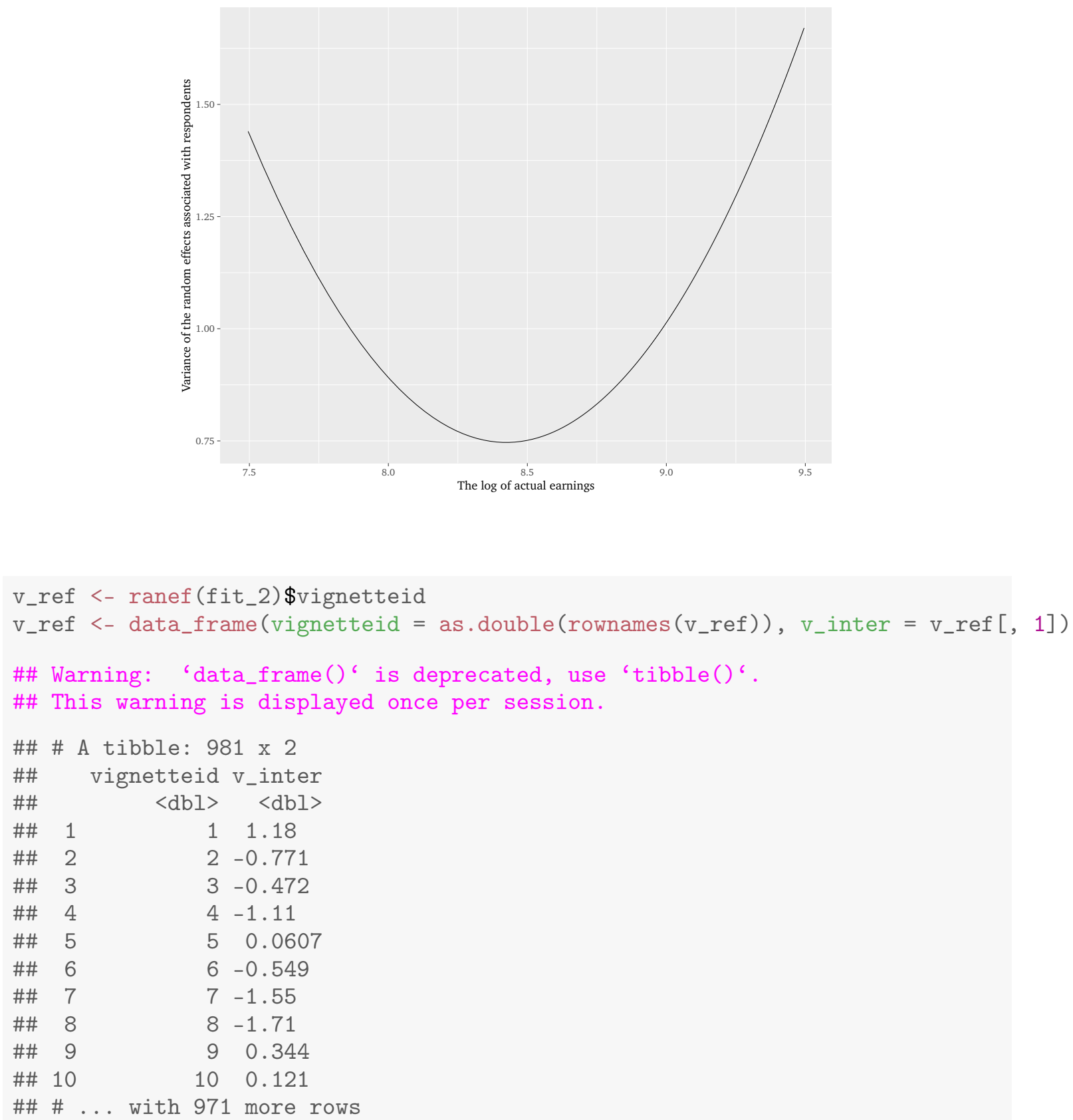

The random effects of vignettes are "stored" in a data-frame object named v_ref that has two columns: ( $a$ a column named vignetteid which contains identification numbers of the vignettes and 
(b) a column named $\mathrm{v}_{\text {_inter }}$ which contains the estimated random effects associated with vignettes. As we can see from the output above, the random effect for the vignette with ID 1 is 1.18 which means that, all else being equal, the intercept for that vignette is shifted up by 1.18 units on the justice evaluation scale, relative to the fixed-effect estimate of the intercept. Similarly, the random effect for vignette 2 is -0.771 , indicating that, ceteris paribus, the intercept for that vignette is -0.771 lower than the fixed-effects estimate. And so forth... We add the random effects to the original data as follows:

$p<-$ left_join $\left(p, v_{-} r e f\right)$

\#\# Joining, by = c("vignetteid", "v_inter")

Here, $\mathrm{p}$ is the name of the original dataset and the message below the command line tells us that $\mathrm{R}$ used vignetteid the merge both datasets.

In a similar way, we extract from the model the random effects of respondents:

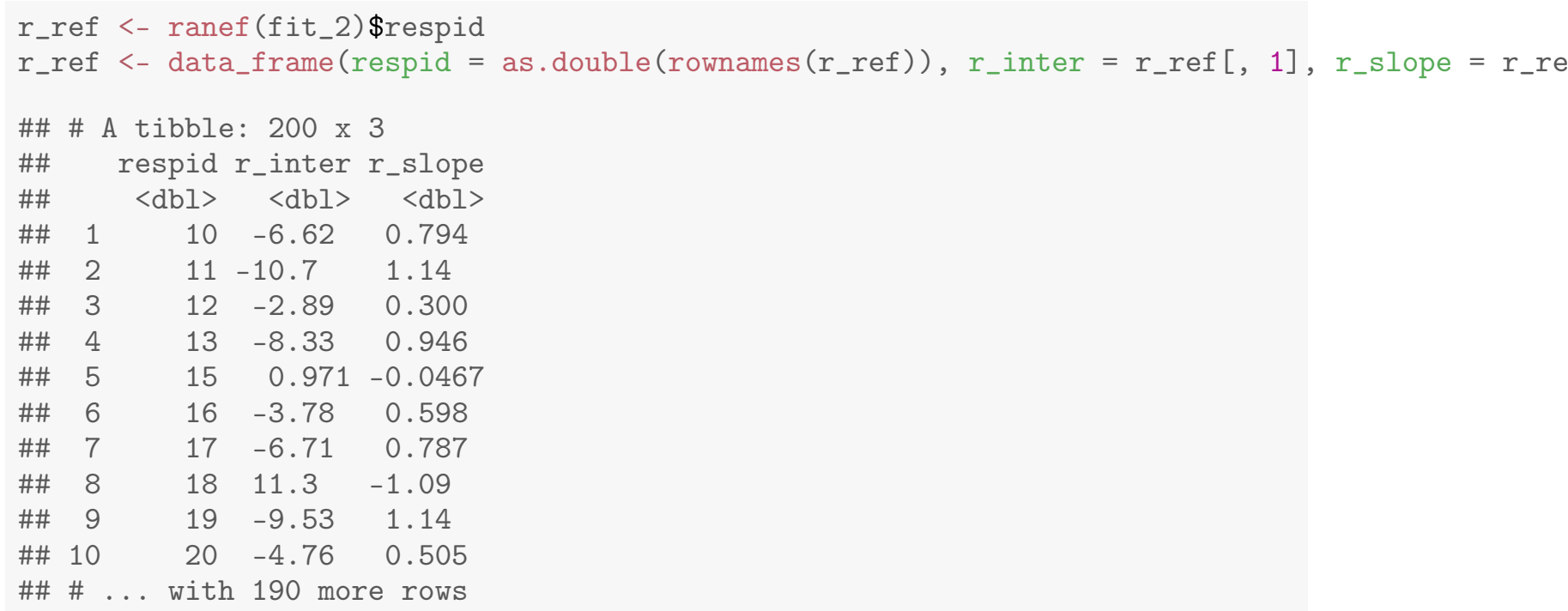

The output above tells us that, other things being equal, the intercept for respondent 10 is -6.62 points below the fixed-effects estimate and that this respondent's slope for the log of actual earnings is 0.794 points larger than the fixed-effects estimate. Merging the random effects of respondents with the original data is performed as follows:

$p<-l_{\text {eft_join( }}$, r_ref)

\#\# Joining, by = c("respid", "r_inter", "r_slope")

Respondent-vignette-specific intercepts are obtained by adding random-intercept effects of respondents and vignettes to the fixed effect estimate: 
$\mathrm{p}<-\mathrm{p} \%>\%$ mutate (beta_0 $=$ fixef(fit_2) [" (Intercept)"] + v_inter + r_inter)

Similarly, respondent-specific slopes are computed as a sum of the fixed-effect estimate of the logged actual earnings and the random-slope effects of respondents:

$\mathrm{p}<-\mathrm{p} \%>$ mutate (beta_1 $=$ fixef(fit_2) $[" \log (\mathrm{pln}) "]+r_{-}$slope)

Finally, grouping the data by respid and vignetteid we are able to create a new data frame that contains the calculated logs of just earnings; we call the new data frame just:

just <- p \% > group_by(respid, vignetteid, r01_gender, r02_age, r03_agecat, r04_region, r05_v

\subsection{Vignette characteristics}

The newly-created dataset just contains information on respondents (stored in variables r01_gender through ret that we can see in the output window above), but it the only information about vignettes - apart from the just earnings - is the vignette identification number. We will use that number now to include vignette characteristics in our data frame.

Earlier on, we mentioned that, when designing our study, we created a population of vignettes by crossing all levels of each characteristic listed earlier with all levels of all the other characteristics, except for job experience (for reasons that we explain below). This resulted in $2 \times 8 \times 21 \times 3=1,008$ unique combinations of the levels of characteristics, each such combination constituting a vignette. From the resulting list, we removed all instances that described a professor with less than 40 years of age, as it is rather rare that a person younger than 40 holds a professorship. Also, while the number of children assigned to vignettes shown to our respondents ranged from 0 to 4 , we actually divided the number of children into three categories: $(a)$ no children, $(b)$ one child, $(c)$ two or more children. This is why we can see 3 levels for children in the formula above. Finally, job experience was not crossed with all other characteristics; instead, it was added to the resulting data set using the following procedure: $(a)$ for each rewardee aged 30, a number from the range 0 to 5 was randomly selected and added to the file as the number of years of job experience; $(b)$ for each rewardee aged 35 , we picked a number from the range 6 through 10; (c) for each rewardee aged 40, we chose a random from the range 11 to 15; (d) for each rewardee.... The "vignette population" is stored in an external CSV file that we now load using the following command:

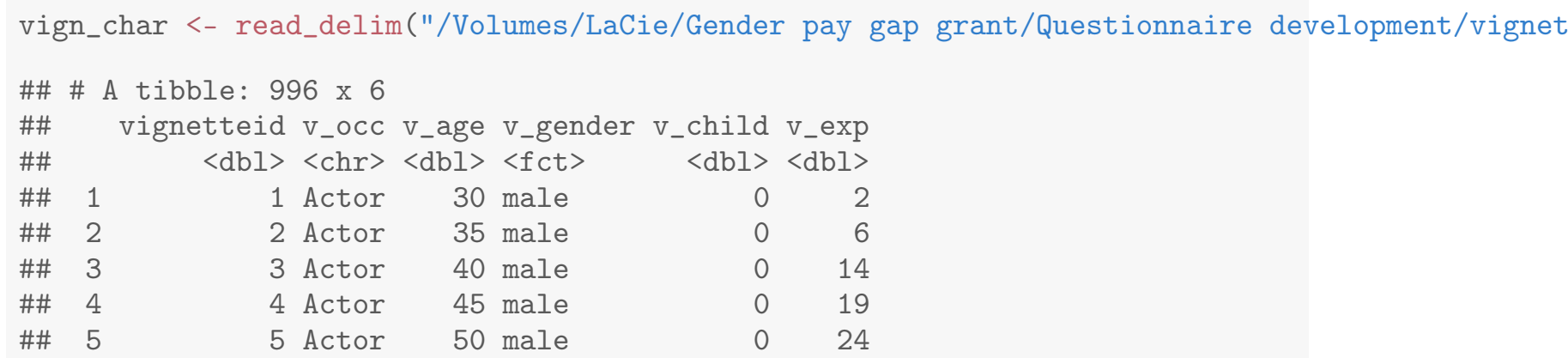


Figure 3: Distribution of the (logged) just earnings by occupation

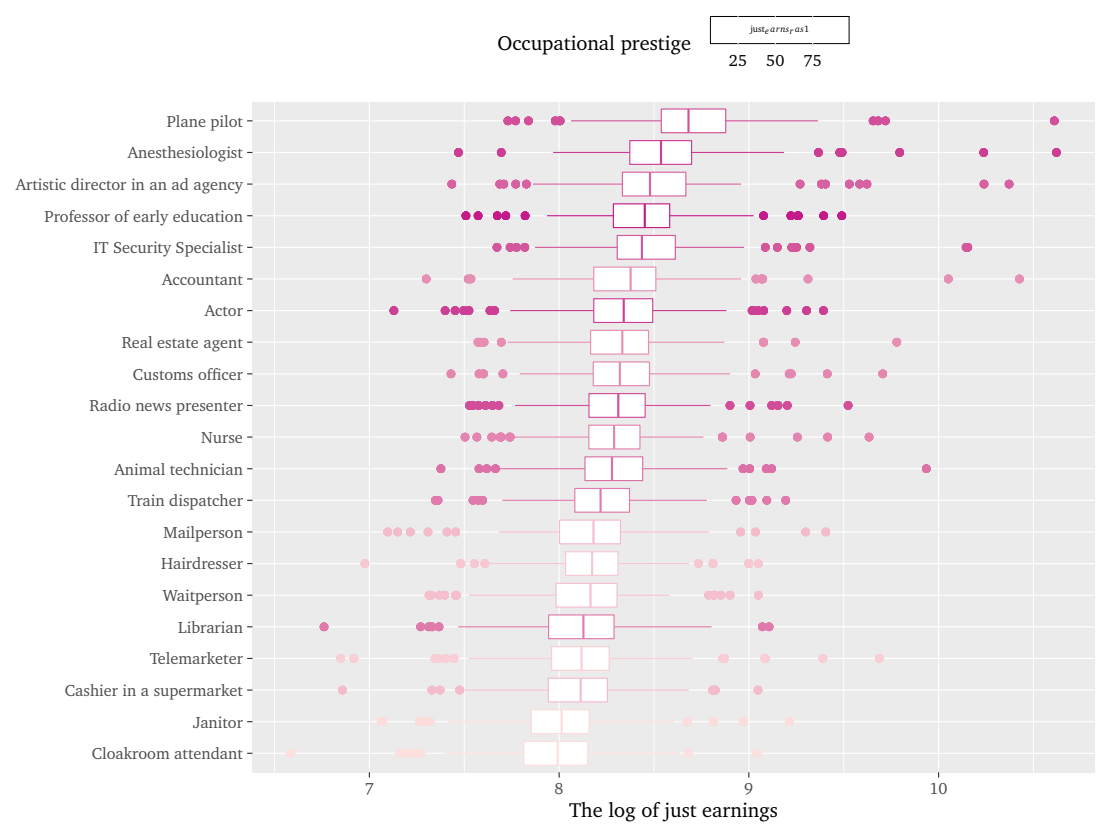

$\begin{array}{rrrrrr}\# \# & 6 & 6 \text { Actor } & 55 \text { male } & 0 & 27 \\ \# \# & 7 & 7 \text { Actor } & 60 \text { male } & 0 & 33 \\ \# \# & 8 & 8 \text { Actor } & 65 \text { male } & 0 & 39 \\ \# \# & 9 & 9 \text { Actor } & 30 \text { male } & 1 & 4 \\ \# \# & 10 & 10 \text { Actor } & 35 \text { male } & 1 & 9 \\ \# \# \text { \# .. with } 986 \text { more rows } & & \end{array}$

The output window tells that that the vignette with ID 1 corresponds to a childless male aged 30 working as an actor with 2 years of job experience. We will now merge the vignette characteristics with the data frame containing the just-earnings estimate that we created earlier:

just <- left_join(just, vign_char)

\#\# Joining, by = "vignetteid"

\subsection{The distribution of just earnings}

Finally, let us have a look at the estimated just earnings. In Figure 3, boxplots of just earnings for each of the occupational titles that we used are shown:

We added colours to the graph to differentiate the occupations in terms of prestige. As we can see, there is a tendency, if imperfect, for more prestigious occupations to be perceived as deserving higher 
earnings. A more detailed analysis of the earnings is a subject of a separate note.

\section{Just earnings and vignette characteristics: A preliminary look}

The estimation of just earnings described above is but an intermediary step in the analysis of just earnings. That is, our ultimate goal is to understand how the amounts of just earnings vary with characteristics of the vignettes and how they vary with characteristics of respondents. These two types of analyses answer different types of questions. That is, looking at the relationship between the just earnings and vignette characteristics is to look at social norms concerning the just distribution of rewards. Further, looking at the relationship between the just amounts and characteristics of respondents is to look at cognitive processes and biases that colour justice perceptions. For instance, if the just earnings vary with respondents' earnings, this may the evidence of anchoring. Finally, there may be interaction between respondents' and vignettes' characteristic, pointing out to possible social identity effects. For instance, if women believe that female workers deserve on average higher salaries than male workers, while men believe that male workers deserve higher incomes than female workers, then it can be interpreted as evidence of in-group favoritism.

In the present section, we provide a rather simple analysis of the relationship of the just earnings that we estimated above and characteristics of the persons described in the vignettes. As mentioned earlier, the descriptions used the following characteristics:

- gender,

- age,

- occupation,

- job experience, and

- the number of children

Note that job experience is measured in years and correlates very closely (at the level of 0.99) with age. This means that we can use only one of the two, as the other provides hardly any additional information, given the strong correlation between them. As for the occupation, to each of the 21 occupational titles that we used in the vignettes corresponds a score on the occupational prestige scale, developed by Domański, Słomczyński, and Sawiński (2009) using the Polish Sociological Classification of Occupations. Instead of using raw scores, however, we divided the occupations into three categories of prestige high, medium, and low - using the following rule: occupations with a prestige score above 70 were classified as "high prestige occupations", occupations with a score below 40 were classified as "low prestige occupations", and all the remaining ones were classified in the medium category. Finally, the number of children was also categories into three classes: no children; 1 child; 2 or more children.

Table 3: Logged just earnings as a function of vignette characteristics

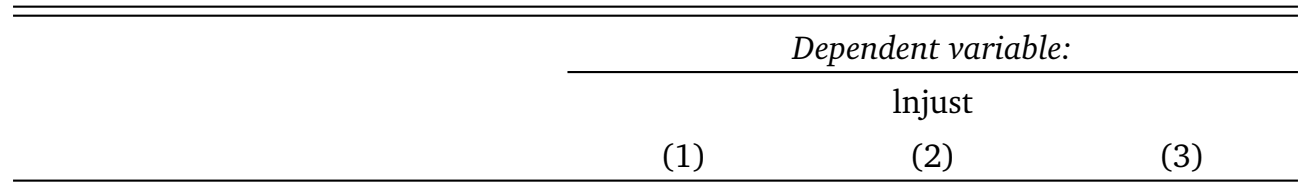




\begin{tabular}{|c|c|c|c|}
\hline Female & $\begin{array}{l}-0.016 \\
(0.011)\end{array}$ & $\begin{array}{l}-0.012 \\
(0.019)\end{array}$ & $\begin{array}{l}-0.005 \\
(0.032)\end{array}$ \\
\hline Age & $\begin{array}{c}0.028^{* * *} \\
(0.004)\end{array}$ & $\begin{array}{c}0.028^{* * *} \\
(0.004)\end{array}$ & $\begin{array}{c}0.028^{* * *} \\
(0.004)\end{array}$ \\
\hline Age sq. & $\begin{array}{c}-0.0002^{* * *} \\
(0.00005)\end{array}$ & $\begin{array}{c}-0.0002^{* * *} \\
(0.00005)\end{array}$ & $\begin{array}{c}-0.0002^{* * *} \\
(0.00005)\end{array}$ \\
\hline Prestige: High & $\begin{array}{c}0.176^{* * *} \\
(0.013)\end{array}$ & $\begin{array}{c}0.176^{* * *} \\
(0.013)\end{array}$ & $\begin{array}{c}0.199^{* * *} \\
(0.032)\end{array}$ \\
\hline Prestige: Low & $\begin{array}{c}-0.179^{* * *} \\
(0.013)\end{array}$ & $\begin{array}{c}-0.179^{* * *} \\
(0.013)\end{array}$ & $\begin{array}{c}-0.168^{* * *} \\
(0.032)\end{array}$ \\
\hline 1 child & $\begin{array}{l}-0.011 \\
(0.013)\end{array}$ & $\begin{array}{l}-0.009 \\
(0.018)\end{array}$ & $\begin{array}{l}-0.012 \\
(0.032)\end{array}$ \\
\hline 2 or more children & $\begin{array}{l}0.027^{* *} \\
(0.013)\end{array}$ & $\begin{array}{c}0.031^{*} \\
(0.018)\end{array}$ & $\begin{array}{c}0.034 \\
(0.032)\end{array}$ \\
\hline Female $X 1$ child & & $\begin{array}{l}-0.004 \\
(0.026)\end{array}$ & $\begin{array}{c}0.019 \\
(0.045)\end{array}$ \\
\hline Female X 2 or more chldrn & & $\begin{array}{l}-0.008 \\
(0.026)\end{array}$ & $\begin{array}{c}0.013 \\
(0.045)\end{array}$ \\
\hline Female X High prst & & & $\begin{array}{l}-0.005 \\
(0.046)\end{array}$ \\
\hline Female X Low prst & & & $\begin{array}{l}-0.015 \\
(0.045)\end{array}$ \\
\hline High prst X 1 child & & & $\begin{array}{c}0.017 \\
(0.045)\end{array}$ \\
\hline Low prst X 1 child & & & $\begin{array}{l}-0.007 \\
(0.045)\end{array}$ \\
\hline High prst X 2 or more chldrn & & & $\begin{array}{l}-0.029 \\
(0.045)\end{array}$ \\
\hline Low prst X 2 or more chldrn & & & $\begin{array}{c}0.019 \\
(0.045)\end{array}$ \\
\hline
\end{tabular}


Fem X High prst X 1 child

\begin{tabular}{lccc}
\hline Observations & 25,200 & 25,200 & 25,200 \\
Log Likelihood & $24,051.470$ & $24,045.930$ & $24,025.270$ \\
Akaike Inf. Crit. & $-48,080.950$ & $-48,065.860$ & $-48,004.540$ \\
Bayesian Inf. Crit. & $-47,991.470$ & $-47,960.110$ & $-47,817.440$ \\
\hline \hline Note: & & ${ }^{*} \mathrm{p}<0.1 ;{ }^{* *} \mathrm{p}<0.05 ;{ }^{* * *} \mathrm{p}<0.01$
\end{tabular}

Before discussing the results of the analysis, let us briefly comment on the analytical approach that we used. Because each respondent evaluated more than one vignette, our observations (i.e., just earnings and corresponding vignette characteristics) can be said to be "nested within respondents". Note, however, that the same vignette was also evaluated by more than one respondent, which means that the observations are "nested within vignettes", too. Because of the nesting, the fundamental assumptions behind the standard regression models are violated. This means that the standard linear regression models cannot be used to study the relationship between variables in our dataset. Therefore, we decided to use mixed-effects linear model, with random effects associated with respondents and vignettes.

A preliminary analysis of the data showed that $7.6 \%$ of the variability in just amounts of earnings can be attributed to differences between respondents and a similar magnitude — ca. $7.5 \%$ - can be attributed to differences between vignettes. Note that the greater the proportion of variance which can be attributed to differences between vignettes indicates the degree of consensus among respondents concerning the just reward for a given vignette.

In Table 3, we show the estimates of parameters for three mixed-effect linear models. For simplicity, we limit the presentation of the results to just fixed effects. Model (1) includes only main effects of the vignette characteristics. Model (2) adds interactions between gender and the number of children. These interaction effects are not statistically significant and they seem to be at odds with hypotheses that we presented in our grant application. For instance, look at the estimate of the parameter for the dummy variable for having 2 or more children in Model (2). As we can see, it is positive which means in this particular case that men with two or more children receive a "fatherhood premium": relative to an otherwise identical man with no children, a father of at least two is believed to deserve a pay $\exp (0.031)=1.031$ times higher. In other words, being a father of two or more children translates into a bonus of about 3\%. For women with two or more children, this bonus is somewhat smaller. To reiterate, this effect is not statistically significant, but it does imply that women with two or more children 


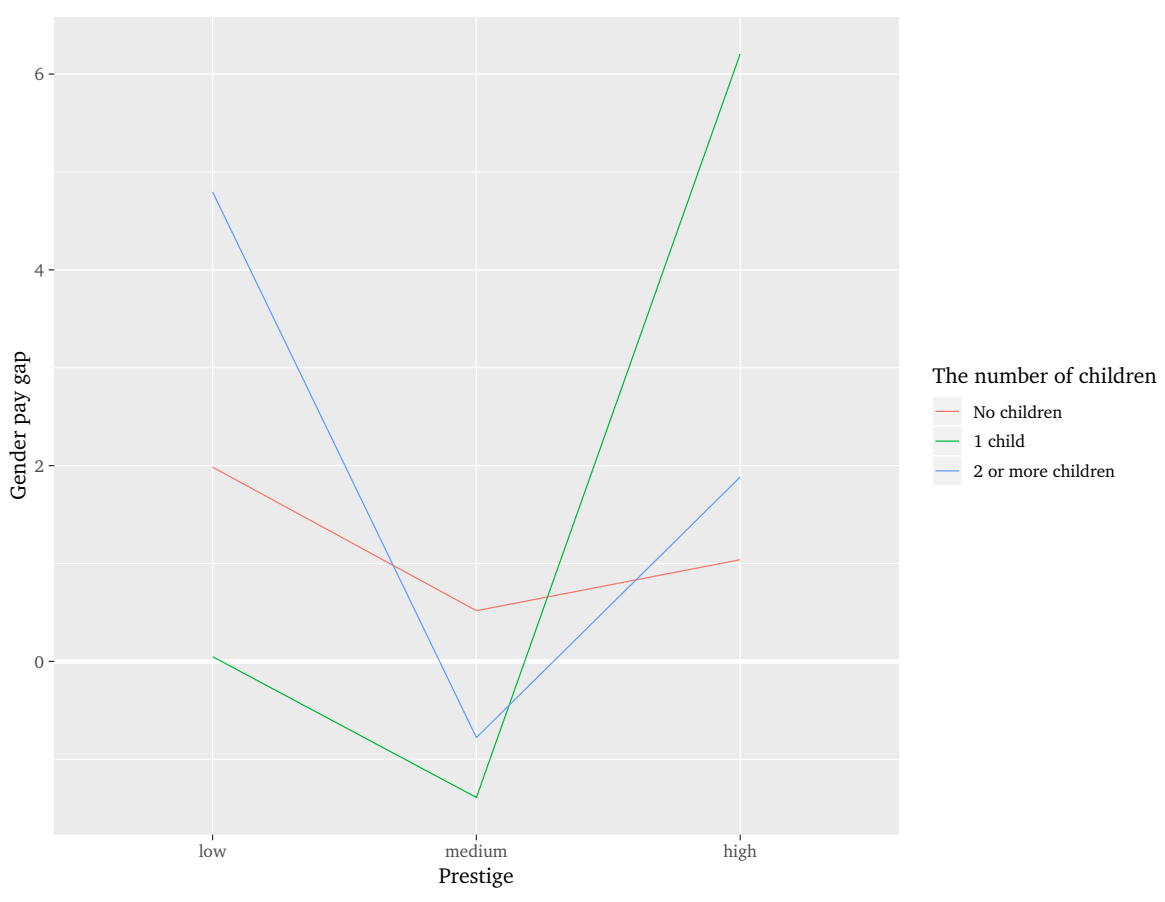

Figure 4: Gender pay gaps by occupational prestige and the number of children

are seen as more deserving than women with no children, which contradicts our theoretical predictions. Model (3) extends the analysis even further by including three-way interactions between gender, the number of children, and occupational prestige. This model was used to test our conjecture that the size of motherhood penalty or fatherhood premium depends on occupational status in the sense that the magnitude of the penalty is greater at higher levels of prestige. The resulting estimates are somewhat consistent with our predictions but still statistically insignificant.

This latter conclusion is confirmed by the graph in Figure 4. The lines in that graph represent sizes of gender pay gaps, where the pay gap is measured in percentages. For instance, a just gender pay gap of $5 \%$ means that women are entitled to earnings $5 \%$ smaller than otherwise identical men according to our respondents. Let us look at the green line which refers to the just gender pay gap for people with at least two children. The pay gap is practically 0 for low-prestige occupations, meaning that in the eyes of our respondent men and women with two or more children who work in low-prestige occupations deserve to be paid the same. But for the high-prestige occupations the pay gap jumps to $6 \%$ meaning that mothers are seen as deserving a pay $6 \%$ lower than fathers of similar characteristics.

\section{Recommendations}

Based on the results of the pilot data, as well as analyses of qualitative responses of pilot participants (not reported here), we make the following recommendations:

1. Structure of the items asking about socio-demographic background of our respondents needs to 
be improved, including the item asking about income.

2. It should be emphasised that the amounts included in the vignettes are net monthly amounts.

3. In case respondent's justice evaluations appeared to be inconsistent or illogical (e.g., a smaller amount is seen as more "appropriate" than a larger amount, but an amount higher than the latter is seen as more appropriate than the former), a warning should pop up asking the respondent to make sure if he or she wants to leave the evaluations as they are or change them.

4. Make the earnings amounts assigned to vignettes more diverse and more spread along the income continuum.

5. If a person described in a vignette is childless, take that information out, so that there is no information about the person's parenthood status, rather than explicit information that he or she is childless.

\section{References}

Bates, Douglas, Martin Mächler, Ben Bolker, and Steve Walker. 2015. "Fitting Linear Mixed-Effects Models Using lme4.” Journal of Statistical Software 67 (1): 1-48. doi:10.18637/ j ss . v067 . i01.

Domański, Henryk, Kazimierz M. Słomczyński, and Zbigniew Sawiński. 2009. Sociological Tools Measuring Occupations: New Classification and Scales. Warsaw: IFiS Publishers.

Eriksson, Kimmo. 2012. "The Accuracy of Mathematical Models of Justice Evaluations." The Journal of Mathematical Sociology 36 (2): 125-135. doi:10.1080/0022250X.2011.556765. eprint: http: // dx.doi .org/10 .1080/0022250x . 2011 .556765. http ://dx.doi .org/10 . $1080 /$ $0022250 \mathrm{X} .2011 .556765$.

Jasso, Guillermina. 2006. "The Theory of Comparison Processes." In Contemporary Social Psychological Theories, edited by Peter J. Burke, 165-193. Stanford, CA: Stanford University Press.

2007. "Studying Justice: Measurement, Estimation, and Analysis of the Actual Reward and the Just Reward." In Distributive and Procedural Justice: Research and Social Applications, edited by Kjell Törnblom and Riel Vermunt. London: Ahsgate.

R Core Team. 2017. R: A Language and Environment for Statistical Computing. Vienna: R Foundation for Statistical Computing. 